\title{
Enfrentamento das doenças cardiovasculares na atenção básica: revisão integrativa da literatura
}

\author{
Coping cardiovascular diseases in primary care: an integrative review
}

Enfermedades cardiovasculares en atención primaria: una revisión integradora

Diorges Boone da Silva ${ }^{1 *}$, André Luiz Freitas Nogueira ${ }^{1}$, Gabriela Tavares Rodrigues ${ }^{1}$, Heriederson Sávio Dias Moura ${ }^{1}$, Anderson Lima Cordeiro da Silva1, Idonilton da Conceição Fernandes ${ }^{2}$, Edney Bentes de Oliveira ${ }^{3}$, Monike Emyline Andrade Rodrigues ${ }^{3}$, Karine Garcêz Mc Comb ${ }^{4}$, Graciana de Sousa Lopes ${ }^{5}$.

\section{RESUMO}

Objetivo: Demonstrar as necessidades de uma promoção em saúde efetiva, frente as doenças cardiovasculares na atenção básica. Métodos: Trata-se de um estudo descritivo, de revisão integrativa, em que se realizou uma busca de produções acadêmicas em periódicos, dando ênfase no enfrentamento das Doenças Cardiovasculares (DCV), utilizando os Descritores em Ciências da Saúde (DeCS): Doenças cardiovasculares; Atenção básica; Fatores de risco e Promoção da saúde. Resultados: Os artigos encontrados, demonstraram que as DCVs, estão entre as principais causas de morte no Brasil e no mundo, sendo possível observar que, os serviços de saúde enfrentam grandes dificuldades em garantir a qualidade de vida das pessoas no enfrentamento à essa enfermidade, por coexistirem vários fatores determinantes para efetivar as necessidades envolvendo uma estratégia de saúde pública efetiva para a comunidade. Considerações finais: A necessidade de combater as DCVs, exige o reconhecimento de que essa enfermidade necessita de um modelo de gestão à saúde que seja eficaz, sendo sustentável dizer que é de grande importância as políticas públicas, seus programas e profissionais capacitados são imprescindíveis para fortalecer medidas cabíveis para o cuidado com as pessoas que se enquadram na DCV.

Palavras-chaves: Atenção básica, Doenças cardiovasculares, Fatores de risco, Promoção da saúde.

\begin{abstract}
Objective: To demonstrate the needs for effective health promotion in the face of cardiovascular diseases in basic care. Methods: This is a descriptive study, of integrative revision, in which a search of academic productions in periodicals was carried out, emphasizing the confrontation of Cardiovascular Diseases (CVD), using the Descriptors in Health Sciences (DeCS): Cardiovascular Diseases; Basic Care; Risk Factors and Health Promotion. Results: The articles found demonstrated that CVD is among the main causes of death in Brazil and in the world. It is possible to observe that health services face great difficulties in ensuring the quality of life of people in facing this disease, because several determining factors coexist to implement the needs involving an effective public health strategy for the community. Final considerations: The need to fight CVD requires the recognition that this disease needs an effective health management model, and it is sustainable to say that public policies, their programs and trained professionals are of great importance to strengthen measures for the care of people who fall into CVD.
\end{abstract}

Keywords: Primary care, Cardiovascular diseases, Risk factors, Health promotion.

${ }^{1}$ Centro Universitário Fametro (CEUNI-FAMETRO), Manaus - AM. *E-mail: diorges248@gmail.com

${ }^{2}$ Universidade Municipal de São Caetano do Sul (USCS), São Caetano do Sul - SP

${ }^{3}$ Singular Educacional (SINGULAR), Manaus - AM

4 Universidade do Estado do Amazonas (UEA), Manaus - AM.

5 Universidade Federal do Amazonas (UFAM), Manaus - AM.

SUBMETIDO EM: 10/2020 


\section{RESUMEN}

Objetivo: Demostrar la necesidad de una promoción efectiva de la salud frente a las enfermedades cardiovasculares en la atención primaria. Métodos: Se trata de un estudio descriptivo, de revisión integradora, en el que se realizó una búsqueda de producciones académicas en revistas, haciendo hincapié en la confrontación de las Enfermedades Cardiovasculares (ECV), utilizando los Descriptores en Ciencias de la Salud (DeCS): Enfermedades cardiovasculares; Atención básica; Factores de riesgo y Promoción de la salud. Resultados: Los artículos encontrados demostraron que las ECV se encuentran entre las principales causas de muerte en el Brasil y en el mundo, y es posible observar que los servicios de salud se enfrentan a grandes dificultades para garantizar la calidad de vida de las personas al hacer frente a esta enfermedad, ya que hay varios factores determinantes para poner en práctica las necesidades que implican una estrategia de salud pública eficaz para la comunidad. Consideraciones finales: La necesidad de combatir las ECV requiere el reconocimiento de que esta enfermedad necesita un modelo de gestión sanitaria eficaz, y es sostenible decir que las políticas públicas, sus programas y los profesionales capacitados son de gran importancia para reforzar las medidas de atención a las personas con ECV.

Palabras clave: Atención primaria, Enfermedades cardiovasculares, Factores de riesgo, Promoción de la salud.

\section{INTRODUÇÃO}

Mundialmente, as doenças cardiovasculares (DCV), são consideradas um desafio para a saúde pública, aumentando de forma progressiva a cada ano. Estas patologias classificam-se como um tipo de doença crônica não transmissíveis (DCNT), as principais complicações são: cardiopatia isquêmica, acidentes vasculares cerebrais (AVC), insuficiência renal crônica (IRC) e insuficiência cardíaca (IC) (SBC, 2010; OPAS, 2011).

O Brasil apresenta uma maior magnitude das DCNT, atingindo todos os níveis socioeconômicos, responsáveis por $72 \%$ das causas de mortes, com destaque para doenças do aparelho circulatório $(31,3 \%)$, câncer $(16,3 \%)$, diabetes $(5,2 \%)$ e doença respiratória crônica $(5,8 \%)$. Sendo que, na grande maioria dos casos diagnosticados, são modificáveis, como: tabaco, alimentação, sedentarismo, etilismo e obesidade (BRASIL, 2011).

Os dados da Sociedade Brasileira de Cardiologia, obtidos no período de 2004 a 2014, denotam que houve um quantitativo de 3.493 .459 óbitos, demonstrando que uma morte a cada 40 segundos ocorre em nosso meio. O ano 2017, foi marcado como o maior período de mortes, ultrapassando todos os anos desde 2004, chegando a 383.961 óbitos por ano, por meio do cardiômetro que é um indicador brasileiro que traz resultados em tempo real, dos números de mortes por DCV (SBC, 2020).

Desta forma, apresentar as doenças do aparelho circulatório, como um problema que devem ser tratadas inicialmente nos serviços da Atenção Básica (AB), por este ser um atendimento primário. Contudo o Sistema Único de Saúde (SUS) enfrenta uma diversidade de problemas que podem ser sugestivos de uma má qualidade neste tipo de atenção primária, podendo citar um deles como o financiamento insuficiente, fazendo com que o enfrentamento das DCV na AB fique deficitário. Isso demonstra que os profissionais envolvidos neste processo, desempenham um papel grandioso enfrentando uma gama de adversidades para prestar serviços com qualidade, enfrentam vários desafios que interferem na qualidade dos serviços (BRASIL, 2013).

Os serviços da $A B$ têm maior poder de descentralização, possibilitando a realização de ações coletivas de promoção e prevenção, podendo suprir problemas e necessidades de saúde das pessoas e grupos populacionais, articulando diversos tipos de tecnologias. Contudo, a atenção primaria em saúde (APS) enfrenta dificuldades na implementação dos seus serviços, gerando dificuldades nas potencialidades e entraves no vínculo da pessoa com DCV, visto que, este é um serviço de "porta aberta" capaz de dar respostas "positivas" aos usuários deste que sejam viáveis (BRASIL, 2013; CUNHA KS, 2016).

Savassi LCM (2012), demonstra alguns aspectos que devem ser foco para melhoria da qualidade na AB, como: a ambiência das unidades de saúde; o uso e a disponibilidade de tecnologias adequadas ao trabalho; 
passar uma imagem de confiabilidade e competência técnica por parte dos profissionais e das equipes; ter agilidade no atendimento; a definição das funções e obrigações dos setores e funcionários; e a priorização da satisfação do usuário.

$\mathrm{Na}$ tentativa de melhorar os atendimentos voltados para os problemas cardíacos na $A B$, assim como reduzir o número de hospitalizações, possibilitando o acompanhar e tratar de forma adequada tanto os casos de hipertensão como diabetes, o Ministério da Saúde (MS) criou em 2002 o Sistema de Cadastramento e Acompanhamento de Hipertensos e Diabéticos (HIPERDIA), este plano que reorganiza os serviços primários e cria estratégias por meio de ações educativas, estímulo à realização de atividades físicas, consultas médicas agendadas e entrega de medicamentos (SILVA JVM, et al., 2015).

Diante do cenário epidemiológico das DCV no Brasil e no mundo, faz-se necessário uma melhor compreensão das estratégias de enfrentamento na $A B$, para que possibilite uma melhor qualidade na assistência primária, visto que, nesta porta de entrada, muitas questões que envolvem a saúde são solucionadas, orientadas e monitoradas, bem como os fatores de risco e suas possibilidades de mudanças na aplicabilidade de novas estratégias. Desta forma, objetivou-se fazer uma análise das dificuldades que encontradas na $A B$ sobre a ótica dos problemas cardíacos e suas prevalências, fazendo uma revisão de literatura, possibilitando à observância das propostas que atuem na qualidade de vida, reduzindo os casos de óbitos por DCV no Brasil, proporcionando incentivo para novas pesquisas relacionadas a temática abordada.

\section{MÉTODOS}

Esta pesquisa foi estabelecida por meio de uma revisão integrativa da literatura, de caráter descritivo, utilizou-se esse tipo pesquisa por apresentar a síntese de múltiplos estudos científicos, com ferramentas que utilizam métodos rigorosos de busca e análise de achados. Além disso, possibilita obter conclusões gerais e específica de cada área de estudo, contribuindo para uma melhor disseminação do conhecimento (MENDES EV, et al., 2008).

A busca dos dados ocorreu através de produções acadêmicas em periódicos, utilizando os Descritores em Ciências da Saúde (DeCS): Doenças cardiovasculares; Atenção básica; Fatores de risco e Promoção da saúde. Para o levantamento dos artigos na literatura, foi utilizado as seguintes bases de dados: Literatura Latino-Americana e do Caribe em Ciências da Saúde (LILACS), Medical Literature Analysis and Retrieval Sistem on-line (MEDLINE), e a Scientific Eletronic Library Online (SCIELO).

Este processo envolveu a seleção dos artigos por meio das variáveis: título, autor, ano de publicação, país de origem, título do periódico, objetivos, principais resultados e conclusões. Após a análise dos achados serem finalizadas, realizou-se a leitura na íntegra para melhor direcionar os resultados e discussão, afim de descrever os desafios da $A B$ e o enfrentamento dos profissionais de saúde na promoção fidedigna das DCV e discuti-lo sob o ponto de vista teórico da saúde pública.

No processo de inclusão dos artigos, considerou-se aqueles que correspondiam aos critérios definidos por: Artigos publicados entre 2015 e 2020; Publicações completas e gratuitas em idiomas nas línguas portuguesa, inglesa e espanhola; Artigos que contextualizem a ideia da proposta, bem como os DeCS nas buscas realizadas. Os artigos que foram desconsiderados para o estudo foram definidos a partir de: Artigos repetidos em demais bases de busca; Não se referirem à adultos e Artigos que fujam da temática central da pesquisa.

A triagem foi realizada pelos integrantes da pesquisa chegando ao resultado e desenho do estudo, onde todos realizaram o mesmo processo de inclusão e exclusão dos artigos encontrados nas bases de dados. Através dos DeCS e os critérios citados para busca e seleção, foram encontrados 153 artigos na Scielo, 1.050 na Medline e 3.114 na Lilacs, totalizando 4.317 achados, destes 23 artigos atenderam os critérios elencados para os resultados (Figura 1).

Após a análise, escolheu-se três categorias, para melhor compreensão do assunto e alcance do objetivo deste estudo. A primeira categoria aborda; os desafios da atenção básica junto aos programas voltados para as pessoas com DCV, por seguinte; Dificuldades dos profissionais de instituir a promoção em saúde nas DCV, e por último; O tratamento para o risco das DCV na atenção básica. Assim espera-se que este assunto traga clareza para demonstrar de que forma a saúde primária está sendo eficaz para a proposta apresentada. 
Figura 1 - Fluxograma dos trabalhos encontrados nas bases de dados pesquisadas.

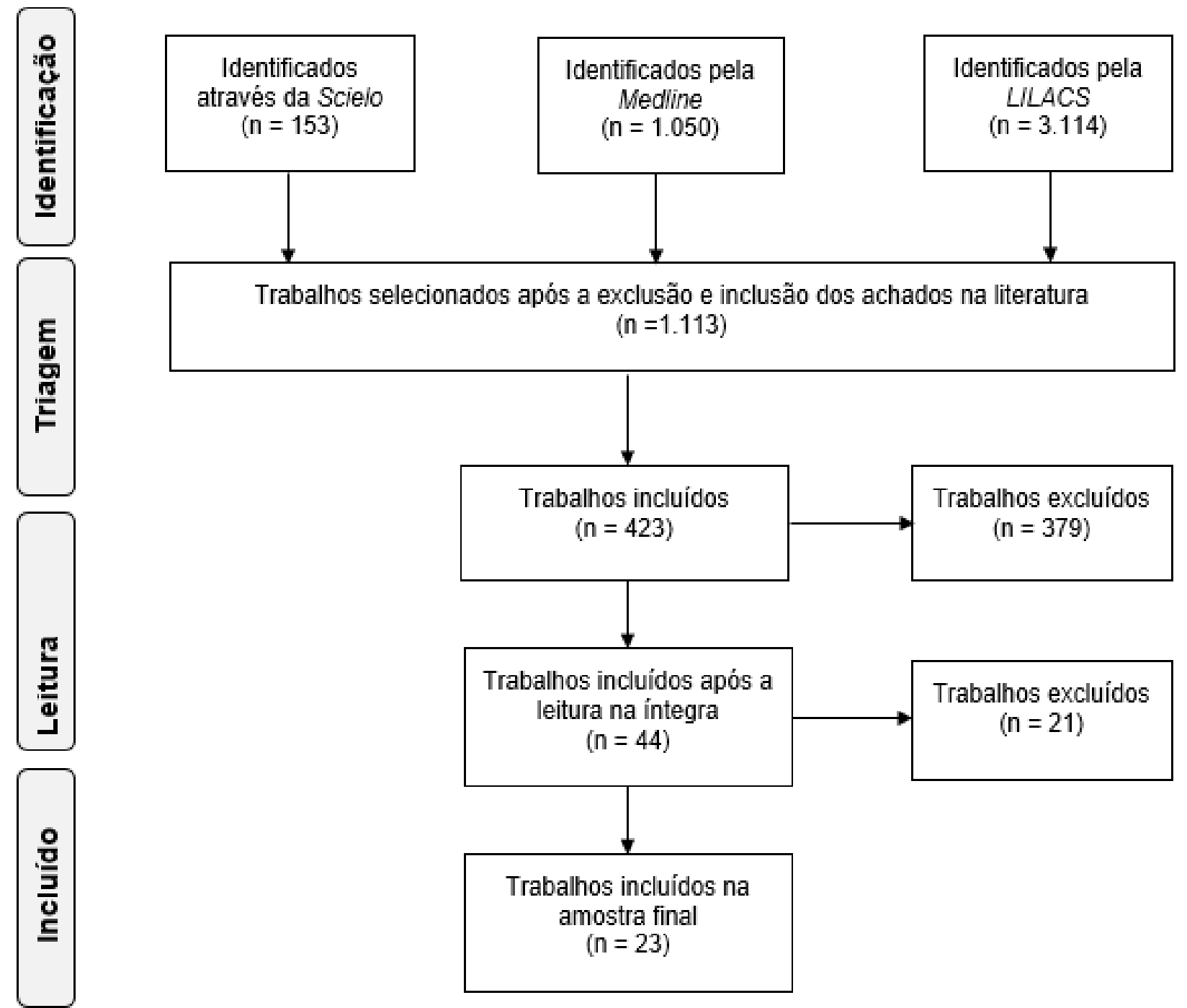

Fonte: Silva DB, et al., 2020.

\section{RESULTADOS E DISCUSSÃO}

O governo brasileiro tem priorizado as DCV, com a implementação das ações previstas no Plano de DCNT 2011/22, destacando os avanços da vigilância e os fatores de riscos (BRASIL, 2011). Mediante aos estudos de Malta DC, et al. (2016), foi observado uma redução na taxa de mortalidade prematura (30-69 anos de idade) por DCNT de quando analisados juntos aos quatro principais grupos de doenças sendo essas: cardiovasculares, câncer, doenças respiratórias crônicas e diabetes, uma redução média de $2,5 \%$ ao ano.

Entretanto, as DCV continuam aumentando exponencialmente, quando comparada com as demais DCNT, Quadro 1, bem como os índices de óbitos no Brasil, mantendo-se ainda a frente das outras DCNT que tiveram maior redução. É de vital importância abordar este assunto devido os números de mortes, visto que é uma doença que apresenta fatores de riscos que podem ser modificáveis, atribuindo a prevenção e mudanças de hábitos que podem fazer com que vidas sejam salvas e que os dados de óbitos possam diminuir.

Segundo Freire AKS, et al. (2017), os estudos demonstram uma baixa produção científica nas áreas da saúde em relação às DCV no Brasil. O que nos faz gerar interesse em aprofundar e elaborar estudos sobre a temática, no que concerne a autoria dos estudos sobre o assunto abordado, nota-se que os profissionais enfermeiros estão a frente com maiores índices de publicações, enfatizando questões sobre os riscos e a importância de uma alimentação saudável. Neste sentido, faz se necessário ampliar produções sobre as ações da promoção à saúde e prevenção da doença. 


\section{Os desafios da atenção básica junto aos programas voltados para as pessoas com doenças cardiovasculares}

A Política Nacional da Atenção Básica (PNAB) foi desenvolvendo, de forma mais marcante e visível na primeira década do século, com intuito de melhorar a assistência prestada na atenção primaria de saúde, viabilizando os programas instaurados que vão de encontro aos problemas de saúde da população, ainda assim, a $A B$ não dispõem de todos os recursos necessários, e de profissionais que contribuam de forma efetiva para o bom funcionamento do que rege os programas (CECILIO LCO e REIS AAC, 2018).

A Hipertensão Arterial Sistêmica (HAS) juntamente com a Diabetes Mellitus (DM) compõem a primeira causa de hospitalizações no sistema público de saúde e são os principais fatores de risco para as DCV, podendo inferir que de 60 a $80 \%$ dos casos, podem ser tratados na AB por meio do programa HiperDia, este sistema gera informações para os profissionais e gestores das Secretarias Municipais, Estaduais e MS, quando são efetivos, reduzem as internações e complicações da doença, assim como traz a chance de uma vida com mais qualidade de saúde (SILVA JVM, et al., 2015).

Os serviços da $A B$, cobrem cerca de $60 \%$ da população brasileira, mesmo com os programas que visam a redução das doenças crônicas, Quadro 1, dentre elas as do aparelho circulatório, os profissionais de saúde enfrentam dificuldades em instituir o que prevê os planos de ação das diretrizes das DCNT, como: Programa Academia da Saúde, Plano Intersetorial de Controle e Prevenção da Obesidade, Atenção domiciliar, que deveriam fortalecer a capacidade de resposta do SUS e à ampliação de um conjunto de intervenções diversificadas capazes de uma abordagem integral da saúde (BRASIL, 2011).

Além disso, otimizar o uso dos recursos em saúde, impede deslocamentos desnecessários e traz maior eficiência e equidade a gestão das listas de espera. O cumprimento dessas funções depende, entretanto, do conhecimento de informações mínimas do paciente para determinar a necessidade da consulta nos serviços ou do procedimento especializado, incluindo a respectiva classificação de risco (BRASIL, 2016).

Uma dificuldade encontrada nos estudos de Dantas RCO e Roncalli AG (2019), foi a falta de insumos necessários para implementar o que prevê os planos e diretrizes impostas para a $A B$ no cuidado aos pacientes com DCV, pois os mesmos precisam de cuidados extensos, avaliações especificas e acompanhamento. $O$ mesmo estudo aponta que os pacientes relatam uma má qualidade nas consultas, e isso dificulta ainda mais a acreditação quanto a adesão fidedigna do tratamento medicamentoso ou não medicamentoso.

Entretanto, a realidade muda de acordo com a região, onde observa-se uma estrutura de atendimento equipada, programas funcionantes de forma integral. Propostas como o Programa Nacional de Melhoria do Acesso e da Qualidade da Atenção Básica (PMAQ) e os Núcleos de Apoio à Saúde da Família (NASF) são respostas positivas, que auxiliam na resolutividade da Estratégia de Saúde da Família (ESF), aumentando o poder de impacto na redução das DCV, desta forma, comprova-se que os municípios com maior cobertura populacional da ESF apresentam uma diminuição da incidência de internações por doenças crônicas, $13 \%$ menores do que os municípios com menor cobertura, sugerindo que a ESF gerou melhorias à saúde da população e desempenho do SUS (BRASIL, 2008; LENTSCK MH e MATHIAS TAF, 2015; PEREIRA MHB, et al., 2018).

Quadro 1 - Fatores de riscos preveníveis para as doenças crônicas não transmissíveis

\begin{tabular}{|c|c|c|c|c|}
\hline DCNT & Tabagismo & Má Alimentação & Sedentarismo & Abuso do álcool \\
\hline Cardiovascular & $\checkmark$ & $\checkmark$ & $\checkmark$ & $\checkmark$ \\
\hline Diabetes & $\checkmark$ & $\checkmark$ & $\checkmark$ & $\checkmark$ \\
\hline Câncer & $\checkmark$ & $\checkmark$ & $\checkmark$ & $\checkmark$ \\
\hline $\begin{array}{c}\text { Doenças Respiratórias } \\
\text { Crônicas }\end{array}$ & $\checkmark$ & $\checkmark$ & $\checkmark$ & $\checkmark$ \\
\hline
\end{tabular}

Fonte: Silva DB, et al., 2020.

De tal forma, denota-se que os programas instituídos para o cuidado as DCV e os fatores de risco que são abordados nos programas são bem discutidos na literatura, como: obesidade, fatores genéticos, idade, sexo, consumo do fumo e/ou álcool, sedentarismo / inatividade física e a má alimentação / dietas inadequadas. 
Diante destes achados, é possível reforçar ainda mais a relação de riscos para DCV modificáveis ou preveníveis para o desenvolvimento das DCNT, entretanto, as produções científicas voltadas para 0 enfrentamento no que concerne a promoção de saúde e prevenção das complicações cardiovasculares, necessitam de uma maior abordagem como mostram os estudos (FREIRE AKS, et al., 2017).

De acordo com Barreto MS, et al. (2018), uma dificuldade da AB está diretamente relacionada com os profissionais de saúde, que em muitos casos podem estar prestando uma assistência em desacordo com os protocolos ministeriais e programas instituídos para estes tipos de cuidados de promoção a saúde, por vezes, estes podem desconhecer os programas para cada tipo de cuidado, levando também a uma limitada explicação ao paciente sobre as formas de acompanhamentos e como proceder para efetivar um tratamento de qualidade, levando a uma baixa utilização dos programas e adesão as consultas, retornos e medicamentos.

Diante de tal problemática, o MS tem fomentado ações, por meio de articulações intersetoriais e interdisciplinares, em conjunto com Plano de Ações Estratégicas para o Enfrentamento das Doenças Crônicas não Transmissíveis no Brasil, 2011/22, porem existem desafios e uma atuação limitada da $A B$ na busca de avanço no cumprimento das metas estabelecidas, assim como a falta de orientação para os programas e para a reabilitação cardiopulmonar (SOUSA CA, 2015; MALTA DC, et al., 2016).

\section{Dificuldades dos profissionais em instituir a promoção em saúde nas doenças cardiovasculares}

O ensejo das propostas de reorientação nos modelos de atenção, geram debates quanto aos modelos biomédicos, visto que houve mudanças do perfil epidemiológico e demográfico da população nas últimas décadas priorizando as práticas de integração do cuidado, medidas de promoção e prevenção. Os profissionais de enfermagem são atuantes em uma diversidade de setores, são estes os facilitadores das mudanças comportamentais de saúde da população brasileira (ALVES DC, et al., 2016).

A equipe de saúde da $A B$, pode atuar de forma significativa, cabendo-Ihes também fazer uma avaliação cardiovascular de qualidade para intervir de forma significativa, aplicando um plano de cuidado individualizado, avaliando a resposta do paciente para que o enfrentamento de uma diversidade de doenças sejam atenuadas, preservando a vida e obtendo uma maior longevidade para as pessoas que sofrem com problemas cardíacos. Portando, é de grande importância reconhecer cada patologia e seus sinais e sintomas para uma identificação precoce e acompanhamento Quadro 2.

Quadro 2 - Sinais e sintomas comuns as pessoas com DCV, e as características de cada sintomatologia.

\begin{tabular}{|c|c|}
\hline Sinais e sintomas & Características relacionadas \\
\hline Dor e desconforto torácico & $\begin{array}{c}\text { Angina de peito, síndrome coronária aguda (SCA), arritmias, } \\
\text { valvopatia cardíaca }\end{array}$ \\
\hline Dispneia & SCA, choque cardiogênico, IC, valvopatia cardíaca. \\
\hline $\begin{array}{l}\text { Edema periférico; ganho de peso; } \\
\text { distensão abdominal }\end{array}$ & IC \\
\hline Palpitações & $\begin{array}{c}\text { Taquicardia secundária, SCA, estimulantes com cafeína ou } \\
\text { outros, desequilíbrios eletrolíticos, estresse, valvopatia cardíaca, } \\
\text { aneurismas ventriculares. }\end{array}$ \\
\hline $\begin{array}{c}\text { Tonturas, síncope ou alterações do } \\
\text { nível de consciência }\end{array}$ & $\begin{array}{l}\text { Choque cardiogênico, distúrbios vasculares cerebrais, arritmias, } \\
\text { hipotensão, hipotensão postural, episódios vasovagal. }\end{array}$ \\
\hline Fadiga vital & $\begin{array}{c}\text { Sintoma de alerta precoce de SCA, IC ou valvopatia cardíaca, } \\
\text { caracterizada por sensação não habitual de cansaço ou fadiga, } \\
\text { irritabilidade ou abatimento. }\end{array}$ \\
\hline
\end{tabular}

Fonte: Silva DB, et al., 2020.

A abordagem inicial do profissional, deve ser ampla, para que todos os sinais possam ser percebidos e orientados de forma adequada para um tratamento fidedigno, ressaltando os cuidados que podem ser atribuídos sem o uso de medicação. Figueiredo DCMM, et al. (2018), corrobora que, as práticas profissionais podem estar intimamente ligadas apenas a queixa e conduta, baseando-se apenas no modelo biomédico, deixando as questões do fortalecimento da autonomia do cliente e suas demais necessidades psicossociais e humanas sem resolutividade ou limitada atenção. 
Observa-se ainda, uma inadequação quanto o direcionamento do cuidado, não estando de acordo com as necessidades e dentro da realidade do contexto socioeconômico, o que leva a uma baixa adesão ao tratamento (FIGUEIREDO DCMM, et al., 2018). O estudo de Dutra DD, et al. (2016), ressalta que a maioria da população desconhece os fatores de risco para DCV, o que evidencia uma preocupação quanto a promoção de saúde adequada, sendo que grande parte da população compreende como fator de risco o consumo de sal e fatores de estresse desconhecendo os demais.

O desenvolvimento profissional, deve ser visto como um processo continuo para a garantia de qualidade da assistência, a carta de Ottawa sobre promoção da Saúde enfatiza de forma clara que o empoderamento de indivíduos são ferramentas importantes sobre o autocuidado, porém, está ainda é uma dificuldade que os gestores encontram na $A B$, podendo ser caracterizado como uma falta de interesse pelos profissionais, falta de incentivos, e ainda a indisponibilidade dos profissionais ou sobre carga de trabalho (CESTARI VRF, et al., 2016).

De acordo com a pesquisa de Maia KAP, et al. (2017), uma dificuldade encontrada na AB que auxiliam na qualidade das assistência, estão evidenciados em medidas simples, como a inadequação dos equipamentos básicos, que são os esfigmomanômetros, onde 78,4\% (264/337) apresentavam mal funcionamento, ainda $52 \%$ (13/25) dos referidos serviços não disponibilizavam manguitos extras para diferentes tamanhos de circunferência de braços, assim como a falta de calibração dos aparelhos, o que dificulta uma boa aferição da PA, o que dificulta um adequado funcionamento das condições diagnosticas.

A abordagem profissional é de grande importância, quando se trata de população com Letramento Funcional em Saúde (LFS) deficiente, sendo de responsabilidade efetiva da equipe de saúde em atribuir uma linguagem efetiva que atenda o nível de conhecimento da população para garantir uma adesão adequada de conhecimento da patologia, dos cuidados e do tratamento (CHEHUEN NJA, et al., 2019).

\section{Tratamento para o risco das doenças cardiovasculares na atenção básica}

As dificuldades de instituir um tratamento de combater a DCV exige o reconhecimento prévio, diagnóstico e direcionamento do cuidado, pois a falta de prevenção, promoção, terapias medicamentosas ou não medicamentosas, quando inadequadas levam a complicações que podem evoluir para o óbito, para isto existem as estratégias de saúde pública que implementa formas de enfrentamento dessas doenças com enfoque no risco (ALVES DC, et al., 2016; AGUAY GJV, et al., 2019).

Para o tratamento dos diversos tipos de DCV, há uma diversidade de fármacos disponíveis, sendo específicos para cada tipo, observa-se uma redução da morbimortalidade cardiovascular quando a adesão ao tratamento farmacológico é eficaz. As intervenções não farmacológicas podem reduzir o uso de medicamentos e ter bons resultados, necessitando de uma boa promoção de saúde. O Brasil tem se mostrado em destaque, frente aos demais países, em relação aos estudos dos efeitos do exercício físico em diferentes condições clínicas Quadro 3 (LIMA DBS, et al., 2016; GOMES MJ, et al., 2019).

Quadro 3 - Modificações de estilo de vida para manejo da HAS e DCV.

\begin{tabular}{|l|l|c|}
\hline \multicolumn{1}{|c|}{ Modificação } & \multicolumn{1}{|c|}{ Recomendação } & Redução da PA em mmHg \\
\hline Redução do peso & $\begin{array}{l}\text { Manter Índice de Massa Corporal (IMC) entre 18,5 } \\
\text { e 24,9 Kg/m² }\end{array}$ & a 20 \\
\hline $\begin{array}{l}\text { Alimentação } \\
\text { saudável }\end{array}$ & $\begin{array}{l}\text { Rica em frutas e vegetais. Pobre em gordura total } \\
\text { e saturada }\end{array}$ & 4 a 9 \\
\hline Atividade física & $\begin{array}{l}\text { Atividade aeróbica, por 30 minutas pelo menos, na } \\
\text { maioria dos dias da semana }\end{array}$ & 2 a 4 \\
\hline $\begin{array}{l}\text { É aconselhável evitar o consumo de bebidas } \\
\text { consumo de álcool }\end{array}$ & $\begin{array}{l}\text { se que o consumo de álcool não ultrapasse 30 ml } \\
\text { de etanol/dia (90 ml de destilados, 300 ml de vinho } \\
\text { ou 720 ml de cerveja), para homens e 15 ml de } \\
\text { etanol para mulheres e indivíduos de baixo peso. }\end{array}$ & \\
\hline
\end{tabular}

Fonte: Silva DB, et al., 2020. 
Para Cardoso NE, et al. (2020), existe uma relação dos fatores de risco para DCV, associando as questões sociodemográficas e clínicas, onde relacionou-se a obesidade ao sexo, estado civil e número de conviventes; sedentarismo, ocupação profissional, idade e número de filhos; o uso de bebida alcoólica, a sexo e ocupação profissional; o consumo de sal ao número de conviventes; e o estresse, à idade, desta forma, podemos analisar que riscos são modificáveis, sendo importantes uma abordagem profissional para que o tratamento não farmacológico voltado para questões educativas, mostrando as medidas preventivas Quadro 3.

Apesar da gama de medicação existentes para tratar os problemas cardiovasculares, a AB dispõem de programas que podem incentivar a adesão às medidas não-farmacológicas, mesmo existente um desafio para instituir tais recursos, essas medidas evolvem envolvem mudanças no estilo de vida, e estão voltadas a minimizar e diminuir a influência dos fatores de risco da vida do paciente, objetivando-se prevenção e controle dessas condições, este estudo contribui com resultados de maior adesão dos portadores de DCNT, como a primeira medida não farmacológica categorizando a atividade física e alimentação saudável em lugar de destaque (PEREIRA MEB, et al., 2016; FREITAS MMA, et al., 2018).

Uma pesquisa realizada por Dantas RCO, et al. (2016), relata a necessidade de programas para rastrear de forma precoce comorbidades como as DCV, incluindo terapêuticas eficazes. Nesta perspectiva, instruir se torna um poderoso modo de munir os sujeitos de informações práticas e diretas, bem como cria relações de confiança entre profissional/usuário, dessa vez, congruentes com as diretrizes políticas da educação em saúde (GAZZINELLIMF, et al., 2015).

Neste viés, é imprescindível a autoavaliação dos resultados dos tratamentos, assim como a melhora das comorbidades associadas, grau de compreensão e aplicação das recomendações na vida diária da pessoa acometida, como os hábitos nutricionais, estado socioeconómico e psicossocial, abrangendo o aspecto geral, desta forma reduzindo as internações e morbimortalidade (SILVA PM, et al., 2019).

\section{CONSIDERAÇÕES FINAIS}

De modo geral, as DCV têm se tornado grandes desafios para saúde pública, gerando preocupação com o dever de cumprir progressos na qualidade de vida, proporcionando medidas de combate e controle desta patologia e seus riscos. É importante que o profissional atribua o cuidado individualizado e direcionado para cada público, para uma melhor compreensão e adesão, visto que a maioria das pessoas que buscam atendimento na atenção primaria são de baixa renda. Baseando-se na pesquisa levantada é sustentável dizer que, as políticas públicas, seus programas e profissionais capacitados são imprescindíveis para fortalecer medidas cabíveis para o cuidado com as pessoas que se enquadram na DCV, por meio de ações de prevenção, acompanhamento e rápido diagnóstico, com intuito de reduzir o acometimento e suas graves consequências.

\section{REFERÊNCIAS}

1. AGUAY GJV, et al. Insuficiencia cardíaca: desafíos en la terapia farmacológica. Revista Digital de Postgrado, 2019. ID: 1094886.

2. ALVES, DC, et al. Promoção da saúde, prevenção de doenças e utilização de serviços: avaliação das ações de uma operadora de plano de saúde brasileira. Cad. saúde colet, 2016. Rio de Janeiro, 24 (2): 153-161.

3. BARRETO MS, et al. Não utilização de consultas de rotina na Atenção Básica por pessoas com hipertensão arterial, Brasil. Ciência \& Saúde Coletiva, 2018; 23: 795-804.

4. BRASIL. Ministério da Saúde. Secretaria de Vigilância em Saúde. Departamento de Análise de Situação de Saúde. Plano de ações estratégicas para o enfrentamento das doenças crônicas não transmissíveis (DCNT) no Brasil 2011 2022 / Ministério da Saúde 2011.

5. BRASIL. Ministério da Saúde. Secretaria de Atenção à Saúde. Departamento de Atenção Básica. Estratégias para o cuidado da pessoa com doença crônica: hipertensão arterial sistêmica / Ministério da Saúde, 2013.

6. CARDOSO NE, et al. Fatores de risco cardiovascular modificáveis em pacientes com hipertensão arterial sistêmica. REME. Rev Min Enferm. 2020, 24:e-1275.

7. CECILIO LCO, REIS AAC. Atenção básica como eixo estruturante do SUS: quando nossos consensos já não bastam! Cad. Saúde Pública. 2018; 34(8):e00136718

8. CESTARI VRF, et al. Competências do enfermeiro na promoção da saúde de indivíduos com cardiopatias crônicas. Rev Bras Enferm. 2016; 69(6):1129-37. 
9. CHEHUEN NJA, et al. Letramento funcional em saúde nos portadores de doenças cardiovasculares crônicas, Brasil. Ciência \& Saúde Coletiva, 2019; 24: 1121-1132.

10. CUNHA KS, et al. Myocardial revascularization: factors intervening in the reference and counter-reference in Primary Health Care. Rev. esc. enferm. USP, São Paulo, 2016, p. 965-972.

11. DANTAS RCO, et al. Determinantes do controle da pressão arterial em homens assistidos na atenção primária à saúde. O Mundo da Saúde, São Paulo - 2016; 40(2):249-256.

12. FIGUEIREDO DCMM, et al. Qualidade do cuidado na Atenção Básica no Brasil: a visão dos usuários. Rev Bras Enferm [Internet. 2018; 40(2):249-256.

13. FREIRE AKS, et al., Panorama no brasil das doenças cardiovasculares dos últimos quatorze anos na perspectiva da promoção à saúde. Revista Saúde e Desenvolvimento. 2017; 11(9).

14. FREITAS MMA, et al. Intervenções não-farmacológicas associadas à prevalência e incidência da hipertensão arterial: uma revisão integrativa. Fisioterapia Brasil. 2018; 19(2).

15. GAZZINELLI MF, et al. Práticas educativas grupais na atenção básica: padrões de interação entre profissionais, usuários e conhecimento. Rev Esc Enferm USP, 2015; 49(2):284-291.

16. GOMES MJ, et al. Non-Pharmacological Treatment of Cardiovascular Disease | Importance of Physical Exercise, Brasil. Arq. Bras. Cardiol., 2019; 113: 9-10.

17. LENTSCK MH e MATHIAS TAF. Internações por doenças cardiovasculares e a cobertura da estratégia saúde da família. Rev. Latino-Am. Enfermagem jul.-ago. 2015; 23(4):611-9.

18. LIMA DBS, et al. Associação entre adesão ao tratamento e tipos de complicações cardiovasculares em pessoas com hipertensão arterial. Texto Contexto Enferm, 2016; 25(3):e0560015.

19. MALTA DC, et al. Avanços do Plano de Ações Estratégicas para o Enfrentamento das Doenças Crônicas não Transmissíveis no Brasil, 2011-2015, Brasil. Epidemiol. Serv. Saúde, 2016; 25: 373-390.

20. PEREIRA MEB, et al. Intervención educativa sobre prevención de cardiopatías congénitas en mujeres en edad fértil, consultorios médicos, Cuba. Ver.Med.Eletrón., 2016; 40: 321-334.

21. SAVASSI LCM. Qualidade em serviços públicos: os desafios da atenção primária, Revista Brasileira de Medicina de Família e Comunidade, 2012; 7(23): 69-74.

22. SILVA PM, et al. Prevalência de fatores de risco cardiovascular e outras comorbilidades em doentes com hipertensão arterial assistidos nos Cuidados de Saúde Primários: estudo Precise, Rev Port Cardiol. 2019; 38(6): 427-437.

23. SOCIEDADE BRASILEIRA DE CARDIOLOGIA. Cardiômetro 2020. Disponível em: <http://www. cardiometro.com.br/anteriores.asp>. Acessado em: 28 de março de 2020.

24. SOCIEDADE BRASILEIRA DE CARDIOLOGIA. VI Diretrizes Brasileiras de Hipertensão, Arq Bras Cardiol., 2010; 95(1): 1-51.

25. SOUZA MT, et al. Revisão integrativa: o que é e como fazer. Einstein. 2010, 8(1): 102-6. 